\section{Kompass \\ Autoimmun}

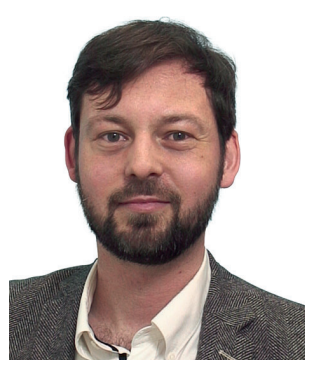

Mike Oliver Becker

Klinik für Rheumatologie,

Universitätsspital Zürich, Zürich, Schweiz

\title{
Diagnostik und Therapie der Vaskulitiden im Wandel
}

Seit Anfang des Milleniums die ersten Berichte über die Behandlung von Anti-neutrophile cytoplasmatische Antikörper (ANCA)-assoziierten Vaskulitiden mit dem B-Zell-depletierenden Antikörper Rituximab erschienen sind, hat dessen Einsatz die Behandlung der Kleingefäßvaskulitiden revolutioniert. Dies gilt nicht nur für die ANCA-assoziierten Vaskulitiden, sondern auch für andere primäre oder sekundäre Kleingefäßvaskulitiden wie z.B. die kryoglobulinämische Vaskulitis oder Vaskulitiden assoziiert mit einer rheumatoiden Arthritis.

Zuvor stand neben klassischen Disease Modifying Anti-Rheumatic Drugs (DMARDs) und Glukokortikoiden vor allem Cyclophosphamid zur Verfügung, welches zwar wirksam ist, aber vor allem in höheren kumulativen Dosen deutliche Nebenwirkungen hat. Demgegenüber geht es heutzutage darum, herauszufinden, was die richtige Therapiesequenz z.B. für lebensbedrohliche Manifestationen einer ANCA-assoziierten Vaskulitis ist wie im Artikel von Kant et. al. beschrieben ([1], Wissenstransfer von Frau Prof. Dr. von Vietinghoff). Die Studien mit dem Komplement-Rezeptor-Inhibitor Avacopan versprechen eine weitere Reduktion der Glukokortikoide auch bei den ANCA-assoziierten Vaskulitiden in der Zu- kunft, sowohl für die Induktions- als auch für die Erhaltungstherapie.

Für andere Entitäten wie die Pupura Schönlein-Henoch bzw. die IgA-Vaskulitis ist der therapeutische Nutzen des Rituximab nicht so klar bewiesen. Hier ist zum einen die Beziehung zwischen IgA-Vaskulitis und IgANephropathie nicht restlos geklärt, zum anderen scheint sich erfreulicherweise die klinische Präsentation der Purpura Schönlein-Henoch im Sinne einer milderen Erkrankung zu verändern ([2], Wissenstransfer von Prof. Dr. Sticherling).

Die Biologika haben inzwischen auch die Therapie der Großgefäßvaskulitiden erreicht, wie die jüngsten EULAR-Empfehlungen zu deren Therapie belegen ([3], Übersichtsarbeit von Prof. Dr. Adler). Dies erlaubt in der Praxis eine deutliche Reduktion der Glukokortikoide und der damit verbundenen Nebenwirkungen. Daneben ist der erfolgreiche Einsatz der bildgebenden Methoden bei der Diagnostik ein weiterer Meilenstein bei der Behandlung dieser Erkrankungen, die auch oligosymptomatisch als inflammatorisches Syndrom auftreten können ([4], Case Report von Poudel et. al. und [3]). Obwohl einerseits noch Forschungsbedarf besteht, z.B. bei der Frage nach der Bildgebung im Verlauf, kündigen sich andererseits mit vielen laufenden klini- schen Studien mögliche weitere Therapieoptionen an, sowohl im Bereich der Biologika als auch der niedermolekularen Verbindungen (small molecules). Damit ist auch hier eine deutliche Verbesserung der Versorgung in der Praxis möglich. Es bleibt abzuwarten, welche der neuen Therapieansätze sich letztlich zusätzlich etablieren werden.

In diesem Sinne wünsche Ihnen viel Spaß beim Lesen dieser Ausgabe von KARGER KOMPASS AUTOIMmUn, welche sich den Vaskulitiden mit Schwerpunkt Großgefäßvaskulitis widmet.

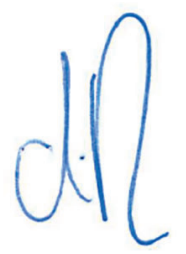

Dr. Mike Oliver Becker

\section{Literatur}

1 Kant S, et al.: Am J Nephrol 2019;50:386-391.

2 Rhim JW, et al.: Clin Rheumatol 2019;38: 2811-2818.

-3 Hellmich B, et al.: Ann Rheum Dis 2020;79: 19-30.

4 Poudel P, et al.: J Investig Med High Impact Case Rep 2019;7:2324709619850222. 